\title{
Mindfulness and dyspareunia: a study of how our mind can dissolve sexual pain
}

\author{
E.A.M. Perez Cruz \\ Faculty of Health Medicine and Life Sciences \\ e.perezcruz@student.maastrichtuniversity.nl
}

\section{Abstract}

Mindfulness has its roots in Buddhism and consists of present moment awareness, attention and acceptance. It is frequently part of a comprehensive treatment protocol, most often cognitive behavioural treatments. This thesis gives an overview on the literature of mindfulness and sexual dysfunctions, dyspareunia in specific, and provides a first step in developing a protocol to test the effectiveness of a mindfulness only intervention. Sufficient evidence has been found in the literature to support the fact that a mindfulness only treatment is indeed effective in treating dyspareunia. Mindfulness decreases the experience of sexual pain during a sexual activity through present moment awareness, attention and acceptance. The findings of this article will lead to further research on the effect of a mindfulness only intervention on dyspareunia and perhaps other sexual dysfunctions and psychological disorders.

\section{Keywords}

Mindfulness, meditation, attention, acceptance, dyspareunia, sexual pain.

\section{Introduction}

Sexual dysfunctions are a considerable problem among many men and women who are sexually active. At least one sexual function disorder is reported by $34.8 \%$ of all men and $43.2 \%$ of all women during their sexually active years. Actual sexual dysfunctions are reported by $12.1 \%$ of men and $22.0 \%$ of women (Buysse et al., 2013). Women report significantly more often sexual problems and dysfunctions than men and they experience more burdens from them. Women have also a vast comorbidity of different sexual dysfunctions (Bakker \& Vanwesenbeeck, 2006). Problems with desire, arousal and pain at the same time are experienced by $75 \%$ of women. The amount of men and women with a sexual disorder or dysfunction who seek professional help for their problems is however 
very low. The reason for this is unknown but it is expected that people are ashamed of their problems or that they are not comfortable talking about such private problems with someone else. Although most people with a sexual problem do not avoid sexual intercourse, $6 \%$ of men and $10 \%$ of women with a disrupted sexual function do. When they have an actual sexual dysfunction these numbers are even higher, respectively $13 \%$ of men and $19 \%$ of women will avoid sexual intercourse (Buysse et al., 2013). Avoidance of sexual activity has a considerable negative effect on the relationship with their partner.

Sexual dysfunctions are thus prevalent disorders with serious consequences for the quality of life of the patient and their partner. Sexual dysfunctions are classified according to the sexual response cycle of Masters and Johnson and Kaplan and can be divided based on desire, arousal, orgasm or pain. This article focuses on the female sexual pain disorder dyspareunia. Dyspareunia is the most prevalent sexual disorder that is reported in clinics.

Dyspareunia is recurrent or persistent genital pain associated with sexual intercourse and causes marked distress or interpersonal difficulty (DSM-IV-TR; American Psychiatric Association (APA), 2000). Thus genital or pelvic pain is experienced before, during or after sexual activity. Dyspareunia is the most common reported complaint women seek help for in hospitals (Gijs et al., 2009). It is mostly experienced by women at an age of eighteen years and younger or at an age of fifty years and older.

There are various treatment possibilities for sexual dysfunctions. Sex therapy techniques are mostly used and consist of sexual education, partner communication skills and sensate focus exercises (Meston \& Bradford, 2007). Cognitive-behavioural therapy is however the main treatment of sexual dysfunctions. It consists of cognitive and behavioural elements wherein inaccurate thoughts and behaviour are challenged through education and exercises. Because the cause of sexual dysfunctions is mostly psychological, cognitivebehavioural therapy is a very effective form of treatment and it is therefore the most practised one.

However, in recent years there has been a shift towards the third wave cognitive behavioural therapies. These consist of acceptance and commitment treatment, behavioural activation, dialectical behavioural therapy, metacognitive therapy, mindfulness-based cognitive therapy and schema therapy. These third wave therapies consist of themes as metacognition, cognitive fusion, emotions, acceptance, mindfulness, dialectics, spirituality and therapeutic relationship (Kahl, Winter \& Schweiger, 2012). They have been proven to be 
effective in the treatment of various psychological disorders and have therefore become increasingly popular.

This thesis will discuss the factor mindfulness of these third wave cognitive behavioural therapies. Mindfulness consists of a present moment awareness which is acquired through meditation, attention and acceptance. Mindfulness integrated in a cognitive behavioural therapy is already been proven to be effective for treating sexual dysfunctions. Sexual dysfunctions are associated with distractions, anxieties, inhibitions, self-criticism about sexual performance and lack of attention to the present moment and on sexual stimuli (Brotto \& Heiman, 2007). Mindfulness can thus be very helpful in dissolving sexual dysfunctions because it focuses on the present moment and not on these distracting thoughts and feelings.

Mindfulness has, however, always been a component of a broader treatment plan, mostly cognitive behavioural therapy. The effectivity of these treatments depends thus on various factors and not just one. It therefore cannot be stated which part is exactly effective; cognitive-based therapy, mindfulness or the combination of these two. This research is therefore going to examine if mindfulness as a treatment only is effective as well in the context of the female sexual pain disorder dyspareunia.

\section{Material and methods}

This research is a preliminary literary study. It examines if a pre-experiment about a mindfulness only intervention for the treatment of dyspareunia has a possible positive outcome. Thirty-four articles about mindfulness, sexual dysfunctions and dyspareunia are used for this literary study. The articles are assembled on PsycINFO and Google Scholar. The used search terms are: mindfulness, dyspareunia, provoked vestibulodynia, sexual pain and sexual dysfunctions.

\section{Results}

Limited research has been carried out on the effects of mindfulness on dyspareunia. Furthermore, there is no research available which has examined the effect of a mindfulness only intervention. This is because mindfulness is a relative new form of treatment in de psychological field. Besides, more occurring and well-known psychological disorders such as depressive and anxiety disorders get more attention. The components of mindfulness, meditation, attention and acceptance, without cognitive-based therapy, are however proven to be successful for treating dyspareunia (Brotto, Basson, Smith, Driscoll \& Sadownik, 2015). Therefore a mindfulness only intervention is expected to be 
effective in the management of dyspareunia. This is because dyspareunia is associated with distractions, anxieties, inhibitions, self-criticism about sexual performance and lack of attention to the present moment and on sexual stimuli (Brotto \& Heiman, 2007). The effect of mindfulness on sexual pain is most likely multifactorial. Mindfulness can increase attention regulation, body awareness, emotion regulation and changes in selfperspective (Brotto, 2013).

\section{Enhanced results}

There is growing evidence that mindfulness is an effective treatment for sexual problems, and dyspareunia in specific. The proven effectivity of mindfulness will therefore be discussed. Mindfulness reduces stress and as a result decreases pain responsivity and this will lead subsequently to less experience of pain. Self-acceptance as a part of acceptance reduces the self-criticism and improves hereby symptoms of dyspareunia (Brotto et al., 2015). Mindfulness reduces pain, distress and pain-related brain activity. Mindfulness can also improve co-morbid anxiety and depression and stimulate sexual arousal. Mindfulness practise increases as well sexual pleasure, arousal and satisfaction because women learn to ignore distracting thoughts and refocus on the sexual activity and correlated stimuli (Brotto et al., 2015). When sexual pleasure, arousal and satisfaction are heightened the perception of genital pain will decrease.

Women with dyspareunia have a low self-efficacy. They view their pain as global and present in all situations, stable and unchangeable and external and uncontrollable (Brotto et al., 2015). A low self-efficacy is a maintaining factor of dyspareunia. Mindfulness increases the self-efficacy of women by challenging the inaccurate biases about their self, their pain and thoughts about sexual avoidance.

Mindfulness reduces pain catastrophizing. People who practice mindfulness ruminate much less about pain (Brotto et al., 2015). The reason for this is the acceptance and present moment awareness which teach people to accept pain and live in the present moment while not worrying about the future. Catastrophizing about pain increases the pain intensity. When the rumination about pain reduces, the fear-avoidance cycle will be broken trough.

Mindfulness encourages women to attend to pleasant and unpleasant physical sensations in the moment instead of being distracted from of overwhelmed by them, and as a result avoid them. Resulting, women experience less genital pain. People who practise 
mindfulness have an increased activity in brain areas which encode sensory aspects of painful stimuli and a decreased activity in areas of appraisal, memory and emotion (Brotto et al., 2015).

Dyspareunia causes conscious avoidance of pain, fear-related inattention to sexual cues and cognitive changes of central sensitization which can impair sexual response and as a result decrease self-acceptance (Brotto et al., 2015). The components of mindfulness, acceptance and attention encourage self-acceptance and as a result improve the sexual response and diminish genital pain. Mindfulness improves self-acceptance because women learn that thoughts are not always truths and they learn to accept themselves.

\section{Discussion/Conclusion}

The goal of this thesis was to provide an overview of the literature on mindfulness and dyspareunia, with the ultimate goal of suggesting a possible protocol to conduct and evaluate a mindfulness only intervention for treating dyspareunia. At this moment, mindfulness is always a part of a greater treatment protocol, which is mostly cognitivebased therapy. The outcome of this research is that a mindfulness only intervention is a potential effective treatment for dyspareunia as well. Mindfulness dissolves dyspareunia through teaching women meditation, attention and acceptance. Meditation, attention and acceptance will cause women with dyspareunia to perceive less pain during a sexual activity through which dyspareunia complaints diminish. Since mindfulness only has a potential positive effect on dyspareunia is it no longer always necessary to merge mindfulness in a cognitive-based therapy. This will have a major impact on the current state of mind in the third wave cognitive and behavioural based therapies. However, this is one of the first studies which examines the effect of a mindfulness only intervention. Much further research has to be carried out to examine the impact of a mindfulness only intervention in the treatment of as well dyspareunia as other psychological disorders.

\section{Limitations and further research}

Mindfulness is founded to be a promising effective treatment for dyspareunia and perhaps other psychological disorders. Despite the fact that mindfulness has already an effect after the first meeting, it is very important to perform mindfulness for at least thirty minutes every day to notice a serious effect. This may be a disadvantage of mindfulness because during the intervention people will be encouraged to do mindfulness every day for at least thirty minutes but once the intervention is over most people will reduce the mindfulness whereby long-term effects will not occur. When people will diminish their 
mindfulness techniques the effect of mindfulness will as well decrease. It is therefore very important to discover a manner to help people stay devoted to their mindfulness techniques.

It would as well be meaningful to design and examine a mindfulness only intervention for dyspareunia where the partner is involved. Because in this intervention, only women participate in a mindfulness only intervention where they acquire mindfulness techniques. This will lead to changes in women which their partners cannot understand because they have not been treated with mindfulness. The women will acquire a new insight regarding to sex but their partners will not share the same opinion. This can lead to an imbalance in the partner dynamics and problems between the partners. These problems can aggravate the complaints of dyspareunia. It will therefore be better to involve the partner in the treatment to prevent problems between the partners.

Perhaps would it be better to treat women with dyspareunia according to the biopsychosocial model where the mindfulness only intervention is one component of the treatment plan. The psychological factors will be handled by mindfulness while the biological determinants of dyspareunia will be treated with surgery or pelvic floor muscle therapy and the social factors with partner and relationship therapy. Mindfulness can be a treatment according to the biopsychosocial model because it can handle the biological, psychological and social determinants together. It will, however, be more responsible to examine the effect of a mindfulness only intervention further before mindfulness only interventions will take care of all determinants at the same time.

This is the first research which examines the effects of a mindfulness only intervention. This is quite odd since mindfulness is a familiar concept in the psychological field for a while now. The reason for this could be that cognitive-based therapy is still the most practised and ruling treatment. People could think that mindfulness has to be a part of a cognitive-based therapy because of the established effectivity of cognitive-based therapy. It is however very important to carry out more researches which examine the effects of a mindfulness only intervention. In this way a mindfulness only intervention could be an established treatment as well. 


\section{Role of the student}

I was under the supervision of Marieke Dewitte who introduced me to the research on the effect of mindfulness on dyspareunia. Since the pre-experiment of the effect of a mindfulness only intervention on dyspareunia was delayed, I carried out a preliminary literary study on this subject on my own. My contribution to the research was that I examined if the pre-experiment had a possible positive outcome. The outcome of my literary study was that a mindfulness only intervention has a positive effect on dyspareunia whereby the pre-experiment has a promising effect.

\section{Acknowledgements}

I thank Marieke Dewitte for introducing me to this specific topic and research and for her guidance during my thesis and the accurate and useful feedback she has given me.

\section{References}

1. American Psychiatric Association. (2000). Diagnostic and Statistical Manual of Mental Disorders, (Fourth Edition, andText revision). DC: Washington

2. Bakker, F., \& Vanwesenbeeck, I. (2006). Seksuele gezondheid in Nederland 2006. Delft: Eburon.

3. Brotto, L. A. (2013). Mindful Sex. Canadian Journal of Human Sexuality, 22, 63-68.

4. Brotto, L. A., Basson, R., Smith, K. B., Driscoll, M., \& Sadownik, L. (2015). Mindfulness-based Group Therapy for Women with Provoked Vestibulodynia. Mindfulness, 6, 417-432.

5. Brotto, L. A., \& Heiman, J. R. (2007). Mindfulness in sex therapy: Applications for women with sexual difficulties following gynecologic cancer. Sexual and Relationship Therapy, 22, 3-11.

6. Buysse, A., Enzlin, P., Lievens, J., T'Sjoen, G., Van Houtte, M., \& Vermeersch, H. (2013). Seksuele gezondheid in Vlaanderen. Gent: Academia Press.

7. Gijs, L., Gianotten, W. L., Vanwesenbeeck, I., \& Weijenborg, P. T. M. (2009). Seksuologie. Houten: Bohn Stafleu van Loghum.

8. Kahl, K. G., Winter, L., \& Schweiger, U. (2012). The third wave of cognitive behavioural therapies: what is new and what is effective? Current opinion in Psychiatry, 25, 522-528.

9. Meston, C. M., \& Bradford,A. (2007). Sexual Dysfunctions in Women. Annual Review of Clinical Psychology,

3. $233-256$. 